\title{
ABDOMINAL ULTRASONOGRAPHY FINDINGS CORRELATED WITH CD4 COUNTS IN ADULT HIV INFECTED PATIENTS
}

\author{
Ashish Kaushik1, Lovely Kaushal2 ${ }^{2}$ Divya Pandey33, Sudeept Dwivedi ${ }^{4}$ \\ 1 Junior Resident, Department of Radiodiagnosis, Gandhi Medical College, Bhopal, Madhya Pradesh. \\ 2 Professor and Head, Department of Radiodiagnosis, Gandhi Medical College, Bhopal, Madhya Pradesh. \\ 3Junior Resident, Department of Radiodiagnosis, Bhopal Medical Center, Bhopal, Madhya Pradesh. \\ ${ }^{4} J u n i o r$ Resident, Department of Radiodiagnosis, Gandhi Medical College, Bhopal, Madhya Pradesh. \\ ABSTRACT
}

\section{BACKGROUND}

The frequency of abdominal disorders in HIV/ AIDS patients is second only to pulmonary diseases. The degree of immunodeficiency is related to the level of CD4+ counts, which is a good index for monitoring the disease progression. It is expected that as the immune status decreases, susceptibility to infection and consequently abnormal sonographic findings should increase.

The aim of the study was to perform abdominal ultrasonography in adult HIV-infected patients and correlate these findings with CD4 counts of these patients.

\section{MATERIALS AND METHODS}

100 adult HIV infected patients were scanned with abdominal ultrasonography and the findings were documented and correlated with their CD4+ counts.

Statistical Analysis Used- Data analysis was done using SPSS 21.0. Two-tailed 'p' values $<0.05$ were considered significant.

Settings and Design- This was a prospective cross-sectional study using sample size of 100 HIV infected patients, conducted at the Department of Radiodiagnosis and Imaging, Gandhi Medical College and Hamidia Hospital, Bhopal from March 2015 to September 2016.

\section{RESULTS}

Splenomegaly, splenic granuloma, focal hypoechoic liver lesions, renomegaly, lymphadenopathy and ascites showed significant correlation with CD4 counts. Incidence of increased renal echogenicity, bowel thickening and hepatomegaly was higher in low CD4 classes, but no statistically significant correlation was found.

\section{CONCLUSION}

Abdominal ultrasonography is an excellent and sensitive modality for routine screening of HIV infected patients and focussed assessment in lower CD4 classes and help in early detection of both infective and neoplastic aetiologies. It can also help in guided FNAC/ Biopsies in suspected cases of neoplasms.

\section{KEYWORDS}

Abdominal Ultrasonography, CD4 Counts, HIV-Infected, AIDS, Splenic Granuloma, Focal Hypoechoic Liver.

HOW TO CITE THIS ARTICLE: Kaushik A, Kaushal L, Pandey D, et al. Abdominal ultrasonography findings correlated with CD4 counts in adult HIV infected patients. J. Evolution Med. Dent. Sci. 2018;7(12):1528-1535, DOI: 10.14260/jemds/2018/346

\section{BACKGROUND}

AIDS (Acquired Immunodeficiency Syndrome) is caused by infection by Human Immunodeficiency Virus (HIV), which belongs to Retrovirus group. Transmission of HIV virus occurs through transfer of blood, semen, vaginal fluid, preejaculate or breast milk.

HIV infects helper T cells (CD4+ T cells), macrophages, and dendritic cells, which play an important role in immune system of human body. HIV leads to low levels of CD4+ T cells through a number of mechanisms. When CD4+ cell count reaches a critical level, cell-mediated immunity is lost and the body becomes progressively more susceptible to opportunistic infections. As a result, various organs of the body may be affected, leading to a variety of clinical presentations together called as AIDS, which is a complex

'Financial or Other Competing Interest': None.

Submission 03-02-2018, Peer Review 03-03-2018,

Acceptance 09-03-2018, Published 19-03-2018.

Corresponding Author:

Dr, Divya Pandey,

Patel Nagar, Sonipat, Haryana.

E-mail: ashish.kaushik96@gmail.com

DOI: $10.14260 /$ jemds $/ 2018 / 346$ life-threatening opportunistic infections and cancers. If not treated mean survival is around 10 years; however, post ART era there has been a significant improvement in survival rate.

HIV infection is a major public health concern worldwide, especially in Indian sub-continent with 2.1 million of HIV infected patients in 2015.1 There is a sharp rise in morbidity and mortality due to HIV/ AIDS with approximately 68,000 HIV related deaths in 2015.1

Though the major target of the HIV virus is the immune system, the frequency of abdominal disorders in HIV/ AIDS patients has been reported to be second only to pulmonary disease. These abdominal manifestations may be on the increase, as the use of antiretroviral therapy has increased life expectancy and improved quality of life.

Radiology plays a crucial role in the management of these patients. Patients with advanced HIV infection referred for radiographic examination of the gastrointestinal (GI) tract have a high incidence and a wide spectrum of abnormal findings.

Radiologic tools (e.g. ultrasonography) serve both diagnostic and interventional roles, and assist in directing appropriate therapy. Computed tomography (CT) and magnetic resonance imaging (MRI) better characterise HIV- 
related abdominal diseases, but are expensive and unavailable in the typically impoverished, HIV-infected Indian subcontinent.

Ultrasonography is an easy to perform, non-invasive, inexpensive and safe imaging technique that is invaluable in India, where AIDS is prevalent and where sophisticated diagnostic tools are not readily available.

The degree of immunodeficiency is related to the level of CD4+ count and as such CD4+ count is a good index for monitoring the disease's progression. It is expected that as immune status decreases, susceptibility to infection and consequently abnormal sonographic findings should increase.

The need for this study lies in the fact that there is relative lack of literature and studies regarding USG findings in HIV infected patients in Indian subcontinent and still less studies correlating these findings with CD4 counts. So our present study is intended to document these ultrasonographic findings in HIV-infected patients and correlate these findings with CD4 counts of these patients.

\section{MATERIALS AND METHODS}

This was a hospital-based, prospective, cross-sectional study done at Department of Radiodiagnosis, Gandhi Medical College and Hamidia Hospital, Bhopal from March 2015 to September 2016 using purposive sampling and a sample size of 100 HIV infected patients with documented CD4+ counts referred to our department for abdominal ultrasonographic assessment.

\section{Ethics}

All subjects were enrolled with detailed oral and written consent. This study was approved by Ethical and Scientific Committee of our Institute.

\section{Inclusion Criteria}

1. Adult patients > 18 years of age with HIV infection confirmed with ELISA.

2. Patients presenting to Radiodiagnosis Department for transabdominal ultrasonography.

\section{Exclusion Criteria}

No exclusion criteria.

\section{Instrumentation and Technique}

All examinations were performed using PHILLIPS HD7XE ultrasonography machine with convex $3-5 \mathrm{MHz}$ array transducer and 11 - $12.5 \mathrm{MHz}$ linear transducer.

To examine the various abdominal organs patients lay on the examination couch in different postures, which included supine, prone, lateral and oblique positions. The abdominal organs are scanned in longitudinal, transverse and oblique planes.

The following criteria was utilised to assess the abdominal organs:

Lymphadenopathy- visualised lymph nodes with the short axis measured;

Hepatomegaly- longitudinal dimension at midclavicular line $>15 \mathrm{~cm}$;

Splenomegaly- longitudinal dimension $>12 \mathrm{~cm}$;
Thickened gallbladder wall- dimension $>3 \mathrm{~mm}$ at the anterior wall;

Pancreatic enlargement- dimensions $>2.5 \mathrm{~cm}, 1.5 \mathrm{~cm}$ or 2.0 $\mathrm{cm}$ for the head, body or tail respectively;

Renomegaly- longitudinal renal dimension $>12 \mathrm{~cm}$;

The grading of renal echogenicity was according to that described by Hricak et al ${ }^{2}$ (grade 0: renal cortical echogenicity < liver parenchymal echogenicity; grade 1: cortex= liver; grade 2 : renal sinus $>$ cortex $>$ liver; grade 3 : renal sinus $=$ cortex $>$ liver .

Thickened bowel wall- wall thickness $>4 \mathrm{~mm}$;

Biliary dilatation- intrahepatic biliary ducts luminal diameter $>2 \mathrm{~mm}$ (or if $>40 \%$ of the diameter of the adjacent portal vein) or extrahepatic biliary duct luminal diameter at the porta hepatis $>5 \mathrm{~mm}$ for patients $<50$ years of age (or 6 additional $1 \mathrm{~mm}$ per additional decade of life).

Grouping into CD4+ classes is done according to the World Health Organisation's (WHO's) classification of CD4+ immunological profile ${ }^{3}$ in adult HIV-infected patients with CD4+ counts $>500 /$ microlitre categorised into the None or Not significant class;

350 - 499 as Mild;

$200-349$ as Advanced;

and a CD $4+$ count $<200$ in the Severe category.

\section{Complete Evaluation of all Patients was done in the following Format}

- Clinical history and examination.

- Laboratory data (HIV-ELISA; CD4+ counts).

- Ultrasonographic evaluation.

\section{Statistical Analysis}

Data analysis was done using SPSS 21.0. Variables were expressed as percentages and comparison was by chi-square analysis. Two-tailed ' $\mathrm{p}$ ' values $<0.05$ were considered significant.

\section{RESULTS}

\section{Ultrasonography Findings/}

Spleen

Splenomegaly (Figure 1) was the most common abnormal finding present in $51 \%$ of patients with maximum proportion of patients in Severe (54.9\%) followed by Advanced (25.4\%), Mild (13.7\%) and least in Not Significant class (5.8\%) (Table 3). Splenic Granulomas (Figure 2) were seen in $23 \%$ of cases with maximum patients in Severe CD4 class followed by Advanced (21.7\%). Both splenomegaly and splenic granulomas showed significant ' $p$ ' values $(0.022$ and 0.044 respectively) suggesting strong correlation between these findings and CD4 counts. Splenic abscess was seen in 1 patient in Severe CD4 class.

\section{Liver}

Hepatomegaly (Figure 3) was present in (50\% cases), out of which maximum patients were in severe CD4 class followed by Advanced CD4 class (Table 3). However, 'p' value was not significant for hepatomegaly. Cause of hepatomegaly was either due to infection, hepatitis, fatty change, focal infective lesions or neoplastic infiltration. Fatty liver was seen in $11 \%$ of cases with maximum cases in severe CD4 class followed by Not Significant CD4 class. Focal hypoechoic lesions were noted in $20 \%$ cases (Table 3) with maximum number of 
patients in severe CD4 class (75\%) followed by Advanced CD4 class with highly significant ' $p$ ' value $(0.031)$ suggesting significant correlation. Focal hypoechoic lesions were noted in $20 \%$ of patients and on follow-up were found to be liver abscess (13) (Figure 4), tubercular granuloma (2) (Figure 5) and fungal granuloma (2) (Figure 6), Kaposi sarcoma (1) (Figure 7) and Lymphomatous infiltration (2) (Figure 8). Diffuse hypoechogenicity and hyperechogenicity of liver were attributed to Hepatitis (Figure 9) and Fatty infiltration respectively. Focal hyperechoic lesions were found to be incidental haemangiomas.

\section{Gall Bladder}

Majority of patients had normal Gall Bladder on Abdominal USG (87\%) (Table 3). Cholelithiasis was seen in $9 \%$ of patients. Oedematous GB wall was seen in $7 \%$ of patients with maximum no. of patients (71\%) in severe CD4 class. GB wall oedema was seen owing to various pathologies like acalculus cholecystitis (Figure 10) and Calculus Cholecystitis, Hepatitis and secondary to Ascites. GB pathologies did not correlate significantly with CD4 counts.

\section{Pancreas}

Pancreas was normal in size and echogenicity in majority of patients (92\%). Bulky Pancreas was seen in $8 \%$ of patients (Table 3). All 8 patients showed altered echogenicity (Hypoechoic) on USG suggesting Pancreatitis (Figure 11). Maximum no. of patients were in severe CD4 class (62\%) followed by advanced CD4 class (25\%) and mild CD4 class (12.5\%). Non-significant CD4 class patients showed no significant sonographic abnormality. There was no significant correlation between pancreas enlargement/ hypoechogenicity and CD4 counts of the patients.

\section{Biliary Tree}

Biliary tree was normal in most of the patients (96\%). 4\% of patients (Table 3) showed dilated intra/extra-hepatic biliary tree; however, no calculus was seen in biliary tree in these patients (Figure 12). Both severe and advanced CD4 class showed 2 patients each (50\%) with dilated biliary tree and mild and non-significant class showed normal biliary tree on ultrasonography. ' $\mathrm{P}$ ' value was 0.458 (No significant correlation between these findings and CD4 counts).

\section{Kidneys}

Kidneys were normal in $61 \%$ of patients. Renomegaly (Figure 13) was the most common abnormal finding (36\%) with maximum proportion of patients in Severe (63.8\%) and Advanced (22.2\%) CD4 classes with significant 'p' value
(0.031) (Table 3). Hyperechoic renal parenchyma (Figure 14) was noted in $29 \%$ of patients and hypoechoic (Figure 15) in one patient. CKD changes (Figure 14) were seen in 3\% of patients. Renal calculi were seen in $16 \%$ of patients. Except renomegaly, there was no significant correlation between ultrasonography findings of kidney and CD4 counts.

\section{Lymphadenopathy}

Enlarged abdominal lymph nodes (Figure 16) were noted in $47 \%$ of patients (Table 3) with maximum proportion in Severe (63.8\%) and Advanced (21.2\%) CD4 class with highly significant ' $p$ ' value of 0.00 suggesting very significant correlation between lymphadenopathy and CD4 counts.

\section{Ascites}

Ascites was present in $29 \%$ of patients (Table 3) with Severe CD4 class having 58.6\% Advanced having 27.5\% and Mild having $13.7 \%$ of patients. Ascites was not seen in Not Significant CD4 class patients. ' $\mathrm{P}$ ' value for Ascites was significant (0.035).

\section{Bowel}

Bowel thickening (Figure 17) was seen in $18 \%$ of patients (Table 3) with maximum no. of patients in Severe CD4 class 61.1\% followed by Advanced (27.7\%) and Mild (11.1\%). Bowel thickening was not seen in Not Significant CD4 class. ' $\mathrm{P}$ ' value for Bowel Thickening was 0.137 (not significant).

\begin{tabular}{|c|c|c|c|c|}
\hline $\begin{array}{c}\text { Age Groups } \\
\text { (Years) }\end{array}$ & Male & Female & Total & $\mathbf{( \% )}$ \\
\hline $18-27$ & $16(61.5 \%)$ & $10(38.4 \%)$ & 26 & 26 \\
\hline $28-37$ & $32(66.6 \%)$ & $16(33.4 \%)$ & 48 & 48 \\
\hline $38-47$ & $8(66.6 \%)$ & $4(33.4 \%)$ & 12 & 12 \\
\hline $48-57$ & $11(91.6 \%)$ & $1(8.2 \%)$ & 12 & 12 \\
\hline $58-67$ & $2(100 \%)$ & $0(0 \%)$ & 2 & 2 \\
\hline Total & $\mathbf{6 9 ( 6 9 \% )}$ & $\mathbf{3 1 ( 3 1 \% )}$ & $\mathbf{1 0 0}$ & $\mathbf{1 0 0}$ \\
\hline
\end{tabular}

Table 1. Age and Sex Wise distribution of the Patients $(N=100)$

\begin{tabular}{|c|c|c|c|}
\hline $\begin{array}{c}\text { CD4+ Class N } \\
\text { (\% Within Class) }\end{array}$ & Normal & Abnormal & Total \\
\hline Not Significant & $3(23 \%)$ & $10(77 \%)$ & $13(13 \%)$ \\
\hline Mild & $4(20 \%)$ & $16(80 \%)$ & $20(20 \%)$ \\
\hline Advanced & $3(12.5 \%)$ & $21(87.5 \%)$ & $24(24 \%)$ \\
\hline Severe & $4(9.3 \%)$ & $39(90.7 \%)$ & $43(43 \%)$ \\
\hline Total & $\mathbf{1 4}(14 \%)$ & $\mathbf{8 6}(\mathbf{8 6 \% )}$ & $\begin{array}{c}\mathbf{1 0 0} \\
(\mathbf{1 0 0} \%)\end{array}$ \\
\hline \multicolumn{3}{|c|}{ Chi-square $=2.321$, p value $=0.509$} \\
\hline Table 2. Normal/ Abnormal Abdominal Ultrasound \\
Scan vs. CD4+ Classification (N=100) \\
\hline
\end{tabular}

\begin{tabular}{|c|c|c|c|c|c|c|c|}
\hline \multirow{2}{*}{$\begin{array}{l}\text { Sonographic } \\
\text { Findings }\end{array}$} & \multirow{2}{*}{$\begin{array}{c}\text { Frequency } \\
(\%)\end{array}$} & \multicolumn{4}{|c|}{ CD4+ Classification N (\%) } & \multirow{2}{*}{$\begin{array}{c}\text { Chi-Square } \\
\chi\end{array}$} & \multirow{2}{*}{$P$ value } \\
\hline & & Not Significant & Mild & Advanced & Severe & & \\
\hline Splenomegaly & $51(51 \%)$ & $3(5.8 \%)$ & $7(13.7 \%)$ & $13(25.4 \%)$ & $28(54.9 \%)$ & 9.630 & $0.022 *$ \\
\hline Hepatomegaly & $50(50 \%)$ & $5(10 \%)$ & $7(14 \%)$ & $13(26 \%)$ & $15(30 \%)$ & 3.799 & 0.284 \\
\hline Enlarged Nodes & $47(47 \%)$ & $2(4.2 \%)$ & $5(10.6 \%)$ & $10(21.2 \%)$ & $30(63.8 \%)$ & 18.324 & $\mathbf{0 . 0 0}^{* * *}$ \\
\hline Renomegaly & $36(36 \%)$ & $2(5.5 \%)$ & $5(13.8 \%)$ & $8(22.2 \%)$ & $23(63.8 \%)$ & 8.857 & $0.031^{*}$ \\
\hline Hyperechoic Kidneys & $29(29 \%)$ & $2(6.8 \%)$ & $4(13.7 \%)$ & $6(20.6 \%)$ & $17(58.6 \%)$ & 4.461 & 0.216 \\
\hline Ascites & $29(29 \%)$ & $0(0 \%)$ & $4(13.7 \%)$ & $8(27.5 \%)$ & $17(58.6 \%)$ & 8.633 & $0.035^{*}$ \\
\hline Splenic Granuloma & $23(23 \%)$ & $0(0 \%)$ & $3(13.0 \%)$ & $5(21.7 \%)$ & $15(65.2 \%)$ & 8.098 & $0.044^{*}$ \\
\hline Hypoechoic Liver (Focal) & $20(20 \%)$ & $0(0 \%)$ & $2(10 \%)$ & $4(20 \%)$ & $14(70 \%)$ & 8.905 & $0.031^{*}$ \\
\hline Renal Calculus & $16(16 \%)$ & $2(12.5 \%)$ & $4(25 \%)$ & $3(18.7 \%)$ & $7(43.7 \%)$ & 0.463 & 0.927 \\
\hline Hyperechoic Liver & $11(11 \%)$ & $3(27.2 \%)$ & $2(18.1 \%)$ & $2(18.1 \%)$ & $4(36.3 \%)$ & 2.258 & 0.521 \\
\hline
\end{tabular}




\begin{tabular}{|c|c|c|c|c|c|c|c|}
\hline (Diffuse) & & & & & & \\
\hline Cholelithiasis & $9(9 \%)$ & $1(11.1 \%)$ & $2(22.2 \%)$ & $2(22.2 \%)$ & $4(44.4 \%)$ & 0.069 & 0.995 \\
\hline $\begin{array}{c}\text { Bulky Hypoechoic } \\
\text { Pancreas }\end{array}$ & $8(8 \%)$ & $0(0 \%)$ & $1(12.5 \%)$ & $2(25 \%)$ & $5(62.5 \%)$ & 2.148 & 0.542 \\
\hline Oedematous GB & $7(7 \%)$ & $0(0 \%)$ & $1(14.2 \%)$ & $1(14.2 \%)$ & $5(71.4 \%)$ & 2.812 & 0.422 \\
\hline Focal Hyperechoic Liver & $4(4 \%)$ & $0(0 \%)$ & $1(25 \%)$ & $1(25 \%)$ & $2(50 \%)$ & 5.253 & 0.154 \\
\hline Dilated Biliary Tree & $4(4 \%)$ & $0(0 \%)$ & $0(0 \%)$ & $2(50 \%)$ & $2(50 \%)$ & 2.596 & 0.458 \\
\hline CKD & $3(3 \%)$ & $0(0 \%)$ & $0(0 \%)$ & $1(33.3 \%)$ & $2(66.6 \%)$ & 1.536 & 0.674 \\
\hline Diffuse Hypoechoic Liver & $2(2 \%)$ & $0(0 \%)$ & $1(50 \%)$ & $1(50 \%)$ & $0(0 \%)$ & 2.636 & 0.451 \\
\hline Splenic Abscess & $1(1 \%)$ & $0(0 \%)$ & $0(0 \%)$ & $0(0 \%)$ & $1(100 \%)$ & 1.339 & 0.720 \\
\hline Hypoechoic Kidney & $1(1 \%)$ & $0(0 \%)$ & $0(0 \%)$ & $0(0 \%)$ & $1(100 \%)$ & 1.339 & 0.720 \\
\hline \multicolumn{7}{|c|}{ Table 3. Sonographic Findings vs. CD4+ Classification (N=100) } \\
\hline
\end{tabular}

*Represents significant ' $p$ ' value and correlation between findings.

The 'p' values are calculated by comparing the sonographic findings in all four CD4 classes and to know whether these findings are occurring by chance or really correlated to low CD4 count. Significant 'p' values mean the increased frequency of that particular finding in that particular class truly correlates to CD4 count and not occurring merely by chance.

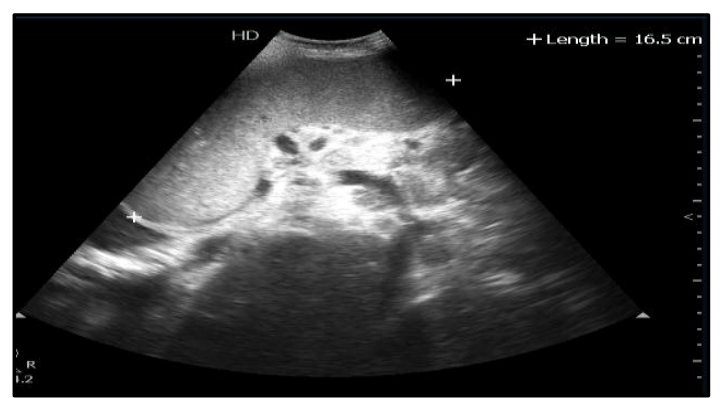

Figure 1. USG Image shows Splenomegaly of $16.5 \mathrm{~cm}$. Left-Sided Pleural Effusion is also Seen

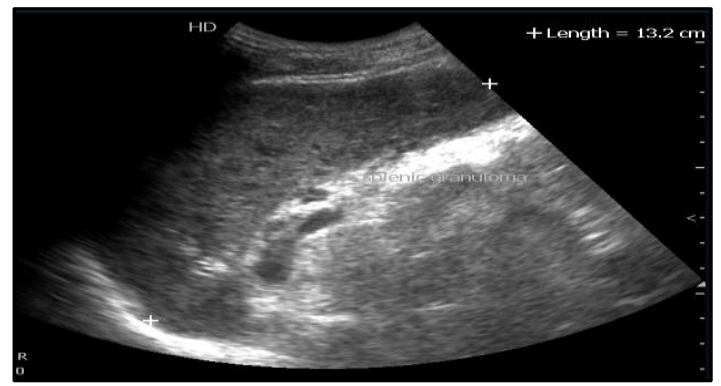

Figure 2. USG Image shows Splenomegaly with Multiple Hypoechoic Lesions (Splenic Granulomas)

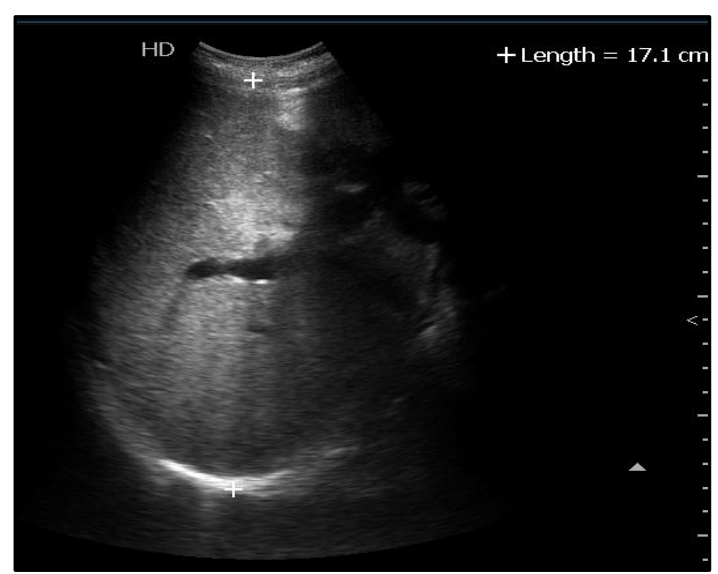

Figure 3. Ultrasound Image of Liver showing Hepatomegaly of $17.1 \mathrm{~cm}$

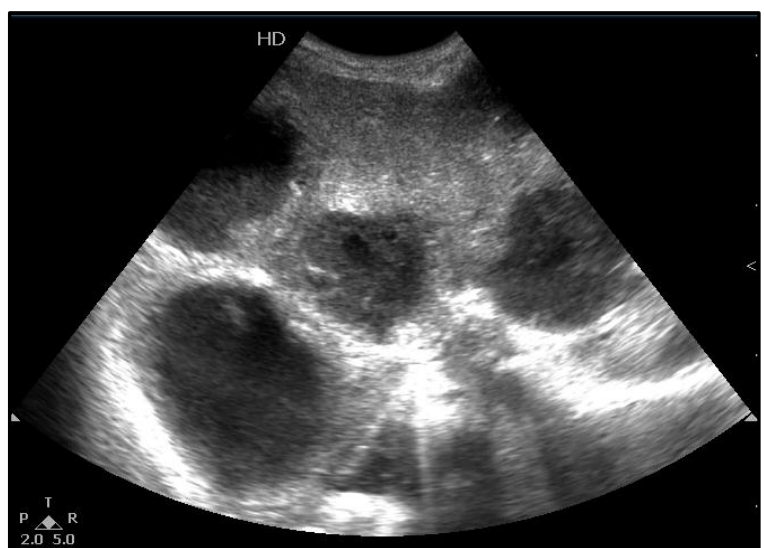

Figure 4. USG Image showing Multiple Hypoechoic Lesions in Liver Parenchyma (Liver Abscesses)

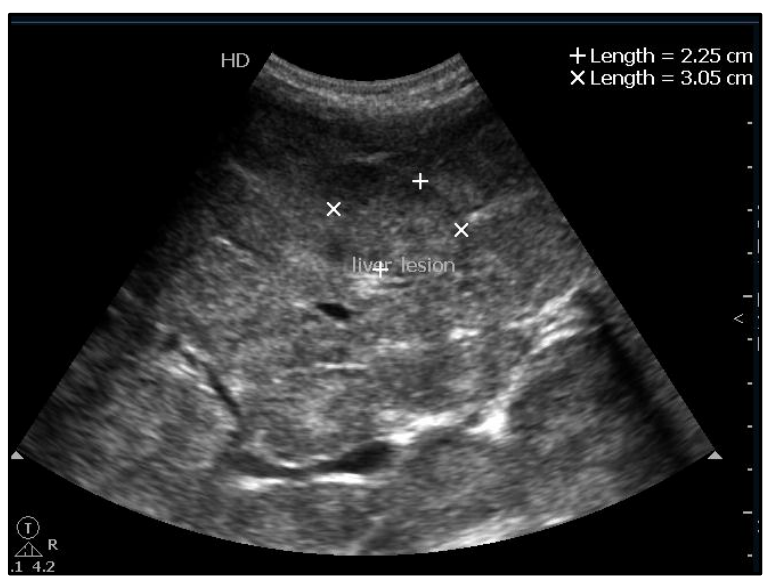

Figure 5. USG Image showing Multiple Round to Oval Heterogeneous Lesions in Liver (Tubercular Granuloma)

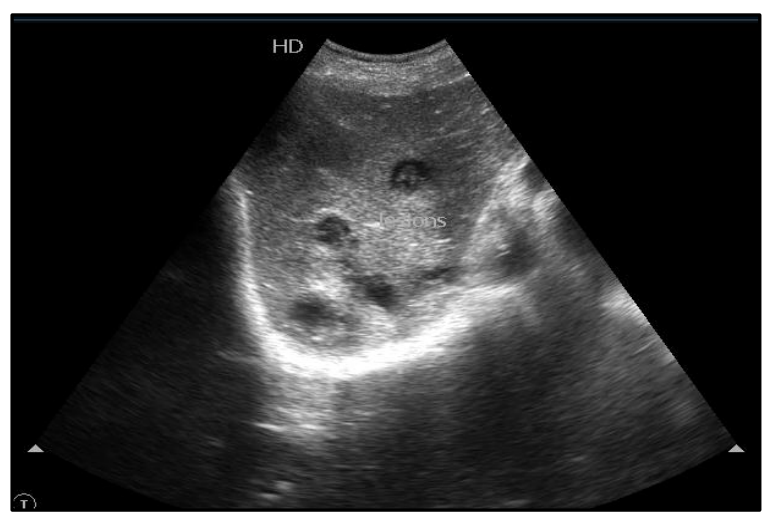

Figure 6. Multiple Bull's Eye Lesions noted in Liver Parenchyma typical of Fungal Granulomas (Candidiasis) 


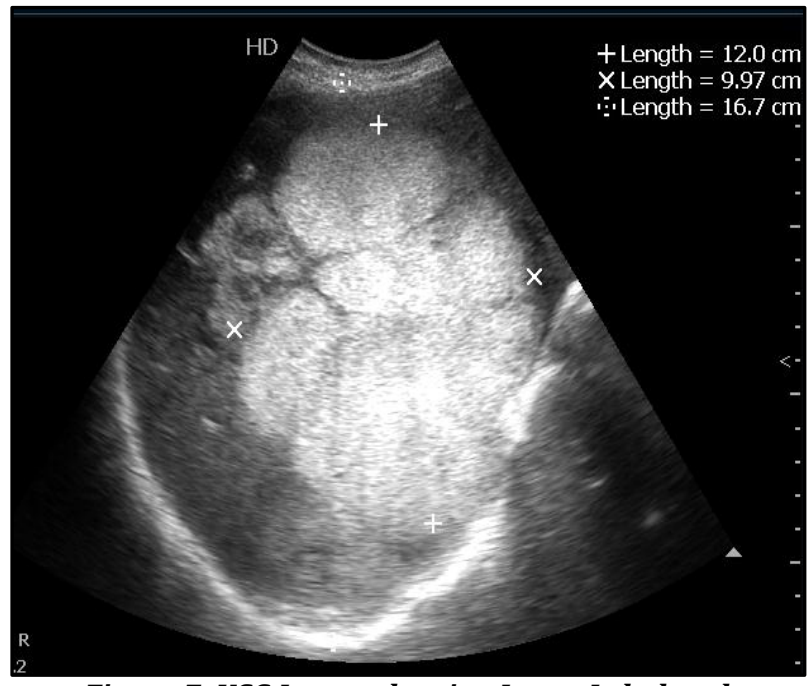

Figure 7. USG Image showing Large Lobulated Hyperechoic Mass (Kaposi Sarcoma)

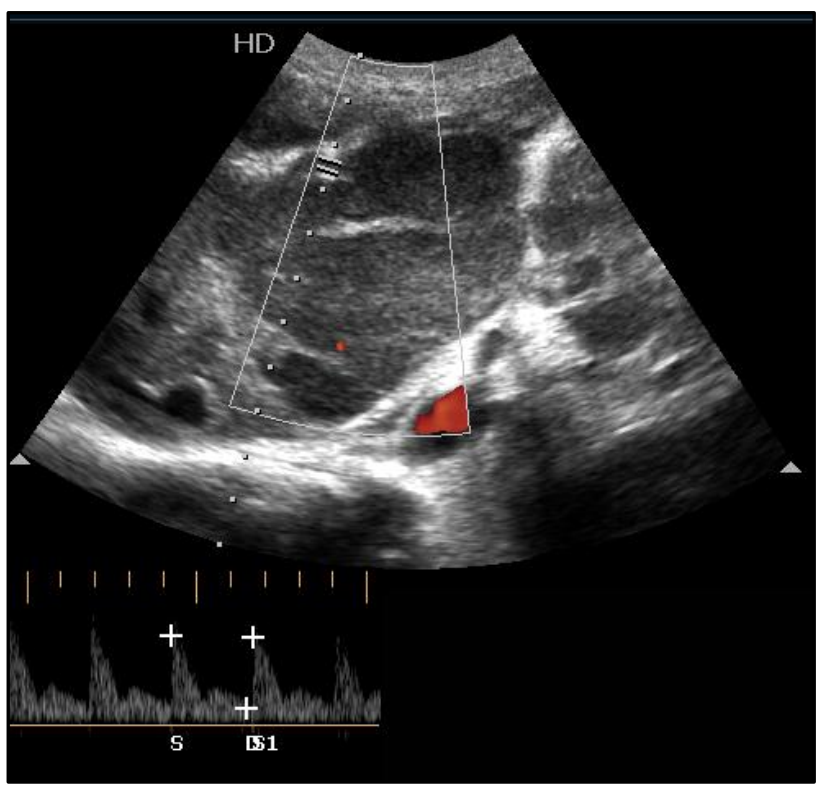

Figure 8. USG Image shows Large Hypoechoic Necrotic Mass Lesion in Left Lobe of Liver showing significant Internal Vascularity, which proved to be Lymphoma on Histopathology

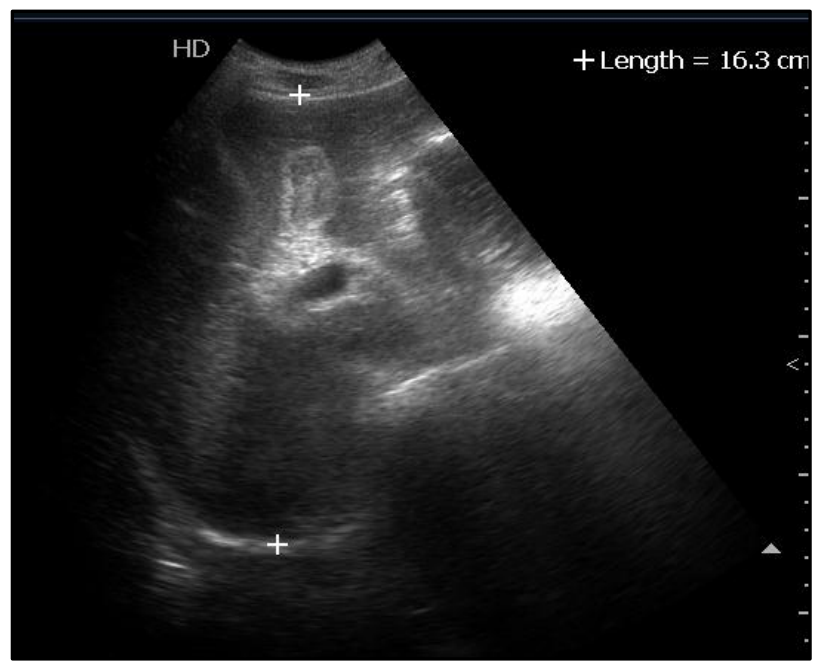

Figure 9. USG Image shows Hepatomegaly with Periportal Cuffing and Increased Echogenicity (Acute Hepatitis)

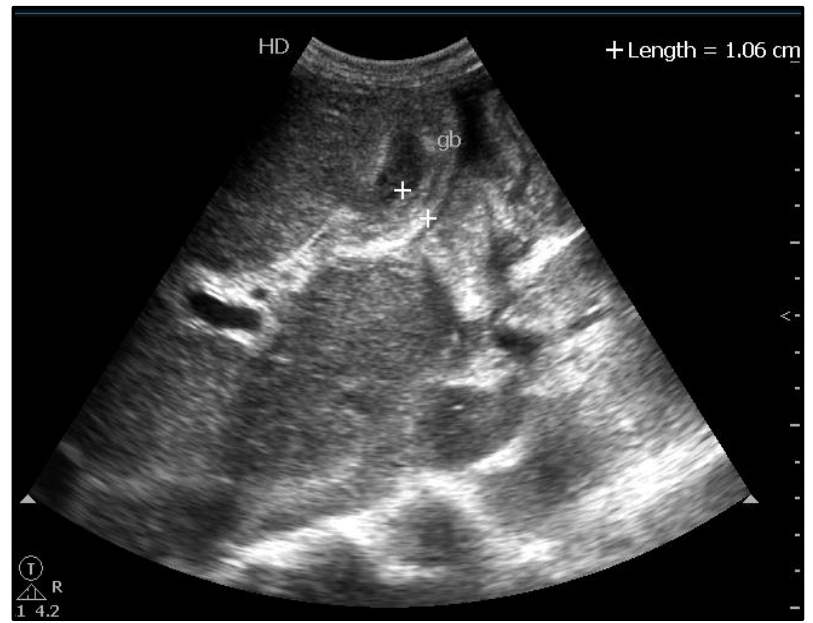

Figure 10. USG Image shows Oedematous GB Wall with Pericholecystic Fluid and Peri-Portal LymphadenopathyAcalculus Cholecystitis

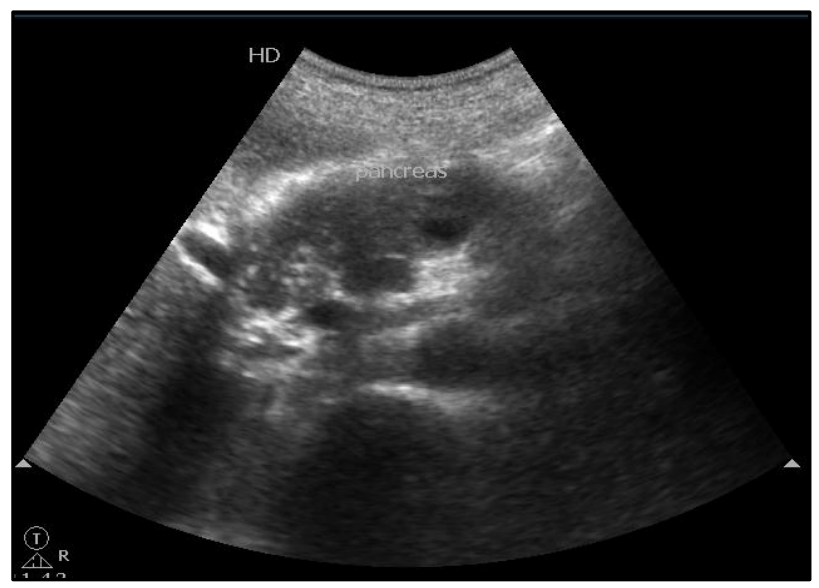

Figure 11. USG Image showing Bulky Hypoechoic Head and Body of Pancreas showing Few Calcific Foci in Head Region suggestive of Pancreatitis

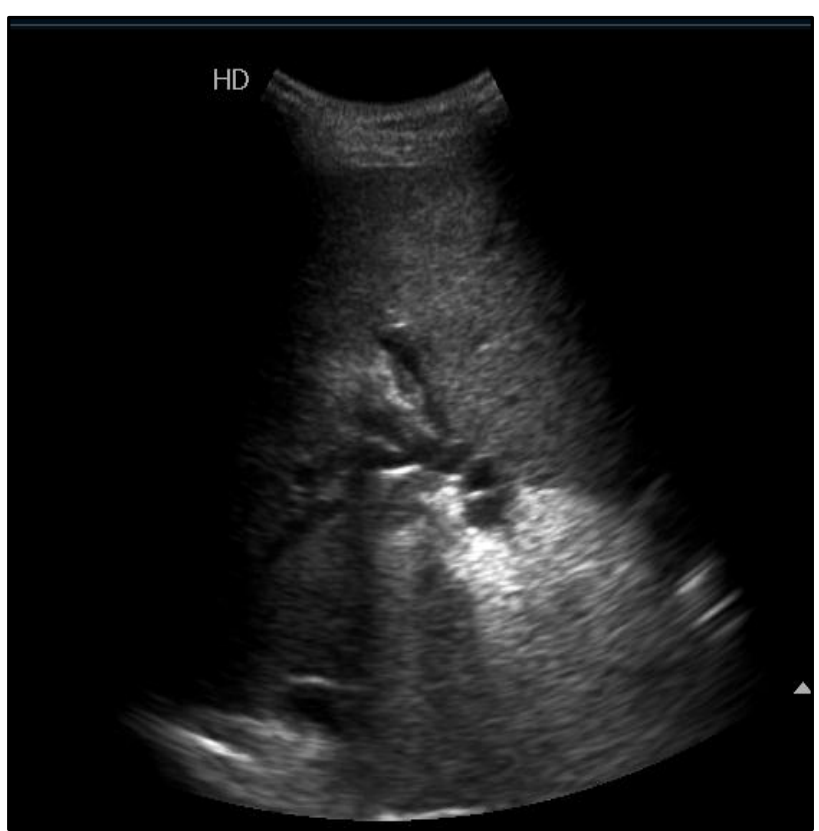

Figure 12. USG Image showing Dilated Central IHBR with Echo Free Lumen (AIDS Cholangiopathy) 


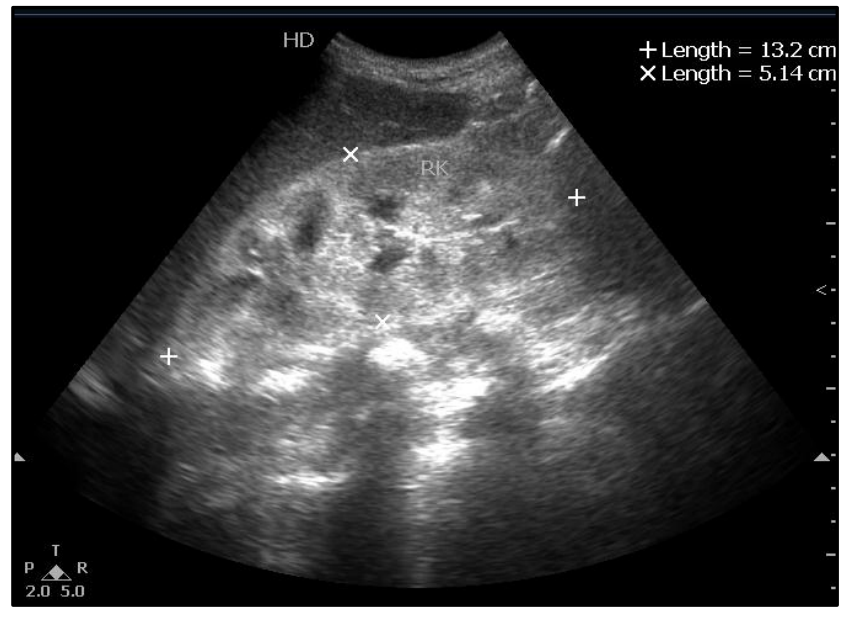

Figure 13. USG Image showing Enlarged Right Kidney with Increased Cortical Echogenicity

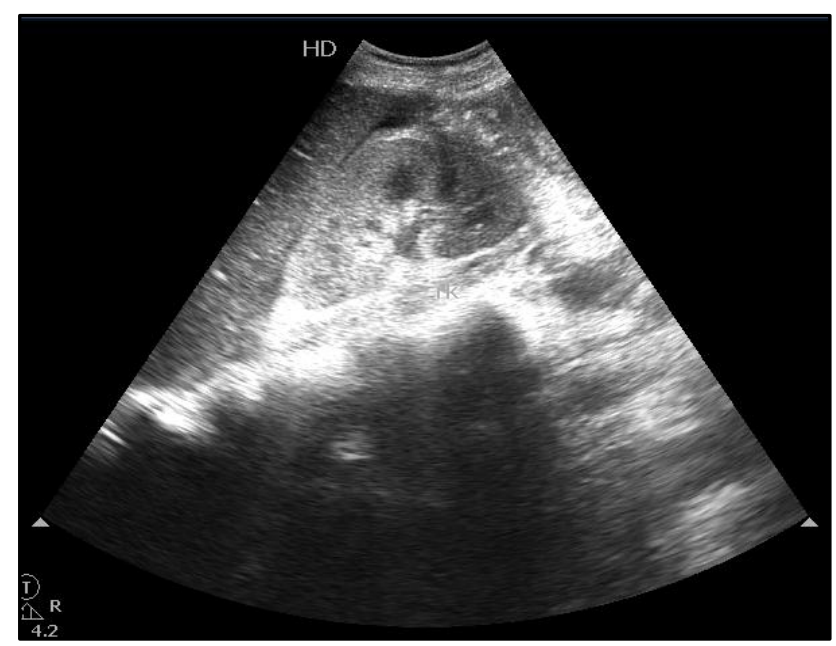

Figure 14. USG Image shows Small-Sized Left Kidney with Increased Echogenicity (HIV Nephropathy)

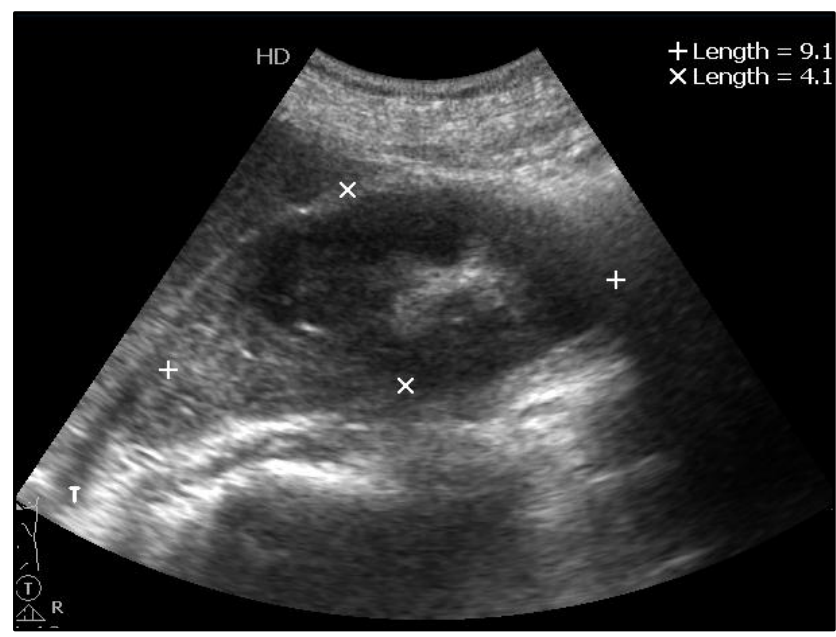

Figure 15. Hypoechoic Lesion in Left Kidney, later Histopathologically proved to be Lymphoma with Renal Involvement

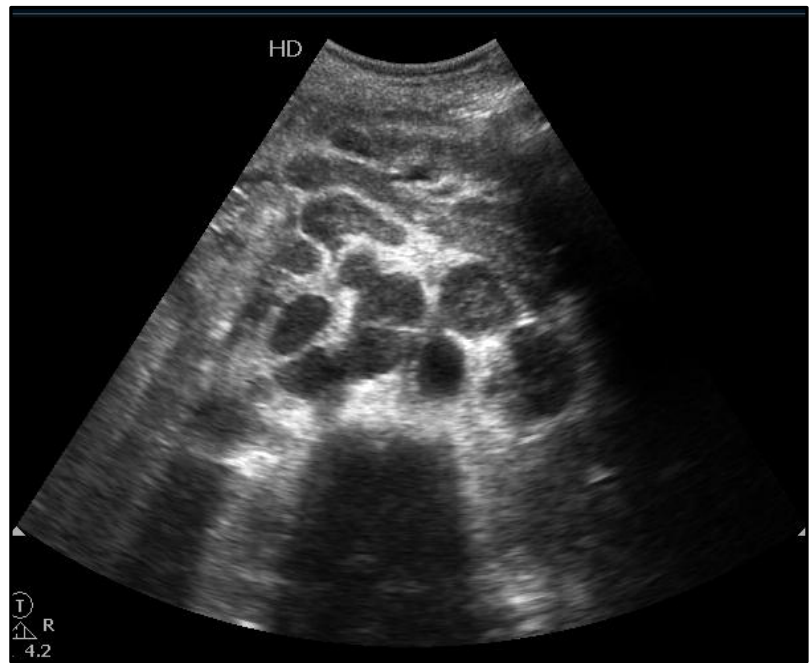

Figure 16. USG Image shows Multiple Enlarged Pre-Para Aortic Lymph Nodes- Lymphadenopathy

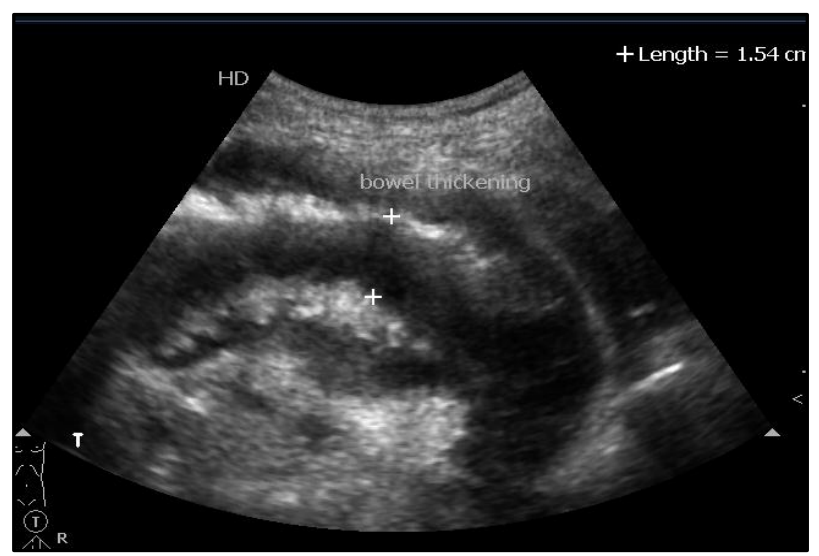

Figure 17. USG Image showing Significant Small Bowel Thickening

\section{DISCUSSION}

Majority of the patients in our study population (around $76 \%$ ) were within the age range of $18-47$ years (Table 1 ). Male patients were far more common in our study (69\%) than females (31\%). This age and sex range nearly corresponds to the data provided by UNAIDS 2015 report, which describes that that AIDS still threatens the cream of society, those in the prime of their working life. ${ }^{4}$

Only $2 \%$ of patients were more than 57 years old, possibly due to the fact that older population are relatively less sexually active. However, with the new ART regimen which provides free drugs and therapy to all HIV infected patients, more elderly HIV-infected patients will be seen in the future due to increased survival rate.

A variety of abnormal abdominal sonographic findings were seen in $86 \%$ of the 100 patients, which is consistent with the observation made by Blessing Ose-Emenim Igbinedion et $\mathrm{al}^{5}$ that out of $300 \mathrm{HIV}$ patients which they reviewed 256 showed sonographic abnormalities in about $85.3 \%$ of the patients. The major sonographic findings included splenomegaly, hepatomegaly, enlarged kidneys, lymphadenopathy, ascites, pancreatitis and bowel thickening. These sonographic abnormalities though are non-specific to a particular pathogen or disease entity, such findings with right clinical correlation and laboratory workup can help the 
treating physicians to arrive at a correct diagnosis which leads to better patient care and treatment.

Most common finding in our study was splenomegaly. The frequency of splenomegaly is comparable with that recorded by Yee et al, 6 in which $45 \%$ of their patients had splenomegaly compared with $51 \%$ in our study. The reason for slightly more incidence of splenomegaly can be malaria, septicaemia, typhoid, schistosomiasis, portal hypertension, haemolytic anaemia and tropical splenomegaly which are quite common in Indian subcontinent. Splenic granulomas presenting as multiple small hypoechoic areas and sometimes small coarse calcifications were seen in $23 \%$ of patients. Both Splenomegaly and Splenic Granulomas showed significant correlation with CD4 counts as both conditions are more prevalent with decreasing immunity status (Decreased CD4 counts) and increased incidence of opportunistic infections (Tuberculosis, Fungal) and Neoplasms (Lymphoma).

Liver showed a variety of sonographic findings with hepatomegaly being most common (50\%), which is consistent with findings of Yee JM et $\mathrm{al}^{6}$ and slightly more than study conducted by Blessing Ose-Emenim Igbinedion et al. ${ }^{5}$ Cause of hepatomegaly was either due to infection, hepatitis, fatty change, focal infective lesions or neoplastic infiltration. Focal hypoechoic lesions were noted in $20 \%$ of patients and on follow-up were found to be liver abscess, tubercular and fungal granulomas, Kaposi sarcoma and Lymphomatous infiltration. Ultrasonography significantly helped in both diagnosis and in few cases guided FNAC/Aspiration of these hypoechoic lesions suggesting its therapeutic advantage also. Diffuse hypoechogenicity and hyperechogenicity of liver were attributed to Hepatitis and Fatty Infiltration respectively. Focal Hyperechoic lesions were found to be incidental Haemangiomas. Focal hypoechoic lesions of liver showed significant correlation with CD4 counts as with decreasing immunity status (Decreased CD4 counts) there is increased incidence of opportunistic infections (tuberculosis, fungal) and neoplasms (Lymphoma/Kaposi sarcoma).

Kidney diseases can be due to HIV virus itself, secondary infections or the administered drugs. Renomegaly showed significant correlation with the CD4 counts and incidence of increased cortical echogenicity was also more in severe CD4 class patients. This statistically significant finding of more patients with renomegaly and associated increased cortical echogenicity may be ascribed to HIV nephropathy, which usually causes diffuse renal cortical echogenicity as well as renal enlargement as suggested by Schaffer et al. ${ }^{7}$ Hypoechoic kidney was attributed to renal involvement by lymphoma. Renal calculus with or without hydronephrosis were seen, but no significant correlation was found with CD4 counts. They may be incidental and may be associated with Indinavir therapy associated crystalluria. These abnormal renal sonographic findings in patients with HIV suggest that even if the patient has no gross evidence of renal disease, a baseline renal sonogram will be useful in patients with lower CD4 counts for prediction and early detection of Renal Parenchymal Disease even before the patient presents with proteinuria or uraemia.

Cholelithiasis noted in few patients was incidental. Acalculus cholecystitis was noted in few patients, which is a well-documented finding in AIDS patients (Nash J et al). ${ }^{8}$ Similarly, dilated biliary tree was seen in two patients which can be due to AIDS Cholangiopathy (Cello J et al). ${ }^{9}$ However, no significant correlation was found in these findings and CD4 counts. Nevertheless, USG is the best modality for assessment of Gall Bladder and Intra/Extra-Hepatic Biliary tree and leads to prompt detection of the above-mentioned pathologies and initiation of timely treatment.

Bulky heterogeneous hypoechoic pancreas noted in 8 patients was attributed to Acute Pancreatitis and correlated with clinical and laboratory findings. Pancreatitis in HIV patients may be owing to toxoplasmosis, cytomegalovirus, M. avium intracellulare or antiretroviral treatment (Stavudine Therapy). Manfredi et $\mathrm{al}^{10}$ in their study found that although the annual incidence in the general population is relatively low, estimated to be 17 to 30 cases per 100,000 population, the annual incidence of acute pancreatitis in the patients with human immunodeficiency virus (HIV) and acquired immune deficiency syndrome (AIDS) is considerably higher.11 No significant correlation was found in these findings and CD4 counts.

Lymph nodes form an important component of the immunity, which acts as a barrier between foreign particles/pathogens and body. In HIV/ AIDS patients, there is increased incidence of Lymphadenopathy due to the increased occurrence of opportunistic infections and neoplasia. In our study, the frequency of occurrence of lymphadenopathy (47\%) was similar to the study conducted by Fabio Cassana et al,12 in which deep nodes were detected in $48 \%$ of HIV infected patients. Lymphadenopathy correlated strongly with patient's CD4+ counts, which can be ascribed to the fact that as patients become more immunecompromised the probability of factors (such as infection and neoplasia) causing lymph node enlargement increases. Enlarged lymph nodes were seen extending from peripancreatic, peri-portal, pre/para-aortic, pre/para-caval, retroperitoneal, mesenteric and omental nodes. Many of the demonstrable lymph nodes had focal hypoechoic areas of necrosis and sometimes coarse calcifications suggesting infective aetiology (either non-specific or tubercular). Large conglomerated nodal mass was seen in 3 patients showing internal vascularity on colour Doppler study and encasement of vessels, which required histopathology and were found to be Lymphoma on follow-up. Detection of these lymphomatous masses further strengthens the credibility of ultrasonography in HIV patients.

Bowel thickening was mainly demonstrated in the small intestine with high incidence in the severe CD4+ class of patients, which may be attributed to the increased frequency of opportunistic gastrointestinal infections with decreased immunity since immunosuppression affects gastric acid secretion and peristalsis (non-specific host defence mechanisms), which predisposes the gastrointestinal tract to increased bacterial colonisation leading to malabsorption, diarrhoea and opportunistic infections. With advent of newer high frequency high resolution probes the rate of detection of bowel pathologies is significantly increasing and it is proving to be excellent screening modality. The incidence of bowel thickening in our study was $18 \%$, which was similar to the study conducted by Tshibwabwa et al, ${ }^{13}$ in which it was $15 \%$ and showed significant ' $p$ ' value (0.001).

Ascites is a non-specific response to many infective and neoplastic aetiologies and its presence suggest the possibility of these pathologies. Ascites was seen in $29 \%$ of patients in 
our study, which is marginally higher than documented by (22\%) Tshibwabwa et al.13 The significant correlation between ascites and CD4 count is due to increased frequency of these opportunistic infections and neoplasms in patients with lower count. Presence of ascites warrants a thorough search for these pathologies and it can be an important clue to the underlying disease.

Although, majority of the above findings are well documented in literature and only few of them correlate well with the CD4 counts. Still there is high incidence and prevalence of these abnormalities in lower CD4 classes owing to the immunological status that makes a certain set of pathologies more common in these patients. And the ability of ultrasonography to detect these findings sometimes in asymptomatic patients warrants more frequent use of sonography in lower CD4 class patients and our present study adds credibility to this statement. These set of patients can be screened at regular intervals owing to low cost and easy availability of sonographic equipment to rule out or early diagnosis of these pathologies, most of which are treatable and will help in increased survival of these patients.

\section{CONCLUSION}

Abdominal ultrasonography is an excellent and sensitive modality for routine screening of HIV infected patients and focussed assessment in lower CD4 classes and help in early detection of both infective and neoplastic aetiologies. It can also help in guided FNAC/ Biopsies in suspected cases of neoplasms.

\section{REFERENCES}

[1] http://www.avert.org/professionals/hiv-aroundworld/asia-pacific/india

[2] Hricak H, Cruz C, Romanski R, et al. Renal parenchymal disease: sonologic-histologic correlation. Radiology 1982;144(1):141-7.

[3] World Health Organization. WHO case definitions of HIV for surveillance and revised clinical staging and immunological classification of HIV-related disease in adults and children. Geneva: World Health Organization, 2006:16.
[4] http://www.unaids.org/sites/default/files/media_ass et/AIDS-by-the-numbers-2016/pg-7

[5] Ose-Emenim IB, Marchie TT, Ogbeide E. Transabdominal ultrasonic findings correlated with CD4+ counts in adult HIV-infected patients in Benin, Nigeria. SA Journal of Radiology 2009;13(2):34-40.

[6] Yee JM, Raghavendra BN, Horii SC, et al. Abdominal sonography in AIDS: a review. J Ultrasound Med 1989;8(12):705-14.

[7] Schaffer RM, Schwartz GE, Becker JA, et al. Renal ultrasound in acquired immune deficiency syndrome. Radiology 1984;153(2):511-3.

[8] Nash JA, Cohen SA. Gallbladder and biliary tract disease in AIDS. Gastroenterol Clin North Am 1997;26(2):323-35.

[9] Cello JP. Acquired immunodeficiency syndrome cholangiopathy: spectrum of disease. Am J Med 1989;86(5):539-46.

[10] Sah RP, Garg P, Saluja AK. Pathogenic mechanisms of acute pancreatitis. Curr Opin Gastroenterol 2012;28(5):507-15.

[11] Manfredi R, Calza L. HIV infection and the pancreas: risk factors and potential management guidelines. Int J STD AIDS 2008;19(2):99-105.

[12] Cassana F, Costigliolab P, Zolia M, et al. Abdominal lymphadenopathy detected by ultrasonography in HIV1 infection: prevalence and significance. Scandinavian Journal of Infectious Diseases 2009;10.3109/00365548.2014.983319.

[13] Tshibwabwa ET, Mwaba P, Bogle-Taylor J, et al. Fouryear study of abdominal ultrasound in 900 Central African adults with AIDS referred for diagnostic imaging. Abdom Imaging 2000;25(3):290-6. 